\title{
Physiological and dynamic response to vibration in cycling: A feasibility study
}

\author{
Marcela Munera $^{a}$, Sebastien Duc, William Bertucci and Xavier Chiementin \\ GRESPI, Université de Reims Champagne-Ardenne, Moulin de la Housse, 51687 Reims Cedex 2, France
}

Received 6 March 2015, Accepted 21 April 2015

\begin{abstract}
Comfort in cycling is related to the level of vibration of the bicycle: more vibration results in less comfort and also it can generate health problems for the rider. The vibration level found in the joints of the lower limb while cycling is directly related to the characteristics of vibration exposure and influenced by the movement in this extremity. The aim of this work was the development of a protocol to analyze the dynamic and physiological response of the human body subjected to vibration exposure while cycling. In this preliminary study, the dynamic response is analyzed using the measure of the transmissibility from the pedal to three joints of the lower body (ankle, knee and hip). In addition, the analysis of the physiological response is done through the measure of muscular activity (electromyography). The subject performs a cycling exercise at a constant speed and power $(80 \mathrm{RPM}, 150 \mathrm{~W})$ with a vibration exposure at six different frequencies (20-30-40-50-60-70 Hz) and with two amplitudes of vibration (2 mm and $4 \mathrm{~mm}$ ). The accelerometric results are analyzed taking into account the different phases of the pedaling cycle. The preliminary results of our analysis show that the vibration frequency and the different phases of the movement have an influence on the induced vibrations. The frequencies and phases where the transmissibility is higher for each joint are found, as well as the frequencies where the muscular activity is increased.
\end{abstract}

Key words: Vibration exposure / cycling / electromyography

\section{Nomenclature}

\begin{tabular}{|c|c|}
\hline$f$ & Frequency \\
\hline$p h$ & Pedaling phase \\
\hline$a_{\text {joint }}$ & RMS acceleration at each joint $\left(\mathrm{m} \cdot \mathrm{s}^{-2}\right)$ \\
\hline$T_{\text {joint }}$ & $\begin{array}{l}\text { Transmissibility to each joint relative } \\
\text { to the input acceleration value }\end{array}$ \\
\hline$a_{\text {input }}$ & RMS acceleration at the pedal $\left(\mathrm{m} \cdot \mathrm{s}^{-2}\right)$ \\
\hline$E M G_{\mathrm{RMS}}$ & $\begin{array}{l}\text { RMS value for the sEMG signals for } \\
\text { each condition of vibration }\end{array}$ \\
\hline$E M G_{-} W V_{\mathrm{RMS}}$ & $\begin{array}{l}\text { RMS value for the sEMG signals } \\
\text { assessed without vibration }\end{array}$ \\
\hline
\end{tabular}

\section{Introduction}

For both professionals and recreational bicycle riders, comfort is important and related to the exercise performance. Comfort in cycling is related to the level of vibration of the bicycle: more vibration results in less comfort for the rider. Vibrations caused by road irregularities are

\footnotetext{
${ }^{a}$ Corresponding author: marcela.munerar@gmail.com
}

transferred throughout the bike to the cyclist. These vibrations can cause health problems mostly associated to the hand-arm system, the knee and the back.

Different studies have quantified the effect of vibration in the human body while cycling. These studies can be divided in two categories: dynamical response and physiological response.

The dynamical response is represented by measures of acceleration at different parts of the human body or at the interface between the body and the bicycle (such as pedals, saddle and handlebar). Using the measures of acceleration the transmissibility between these locations can be also calculated. For the assessment of this dynamic response, different studies take into account the type of bicycle $[1,2]$, its structural characteristics [3,4], the surface roughness $[2,3,5]$, the frequencies of the vibration exposure [6], the speed [3] and the position of the rider.

Some of these studies center their attention on the acceleration at different points of the bike and the transfer function of the frame to the fork [4] or the saddle $[2,7]$.

However, the physical risk associated with this vibration exposure does not depend solely on the vibration at the interface between the rider and the bicycle, but also the vibration at different points of the human body [8]. The transmissibility function to different parts 
of the human body allows the identification of their resonance frequencies. Resonance is the tendency of a system to oscillate at its maximum amplitude, and is associated with specific frequencies so that even small amplitude input vibrations can produce large or dangerous output amplitudes. The identification of resonance is important for determining vibration safety [9] because a higher value of transmissibility could represent a higher risk for each joint. Taking this characteristic into account, in the work of Chiementin et al. [6] the authors propose a study of the transmissibility to the hand-arm system during cycling over a cobblestone road. They found an influence of the speed on the transmissibility and the vibration dose.

The physiological response of the human body to vibration exposure can be measured through different indicators such as the muscular activity (EMG), the heart rate, the oxygen uptake and the muscle metabolism through the production of lactate. In this area, the work of Sperlich and Kleinoeder [10] evaluated the effects of local vibration-induced cycling on the cardiopulmonary system as well as the muscle metabolism and perceived responses compared to cycling without vibration, for power between 250 and $300 \mathrm{~W}$. Their results show that a vibration stimulus leads to a higher respiratory demand. Another study [11], analyzed the performance of dynamic muscular work endurance in cycling under the influence of vibration. This endurance is measured taking into account the time of the exercise, the heart rate and blood pressure. They found that vibrations transmitted to working muscles reduce the physical working capacity measured as endurance during bicycle ergometer exercise at a constant load.

The muscular activity under vibration in cycling has been measured on the forearm muscle [12] and on the back [13], for different road surfaces. However, to the authors knowledge there are not studies that measure the muscular activity at the muscles of the lower limb in cycling under vibration.

In this paper, a new protocol for the analysis of the dynamical and physiological response of the human body to vibrations while cycling is proposed. This response is measured for six frequencies of exposure and two amplitudes. The dynamic response is analyzed through the measure of vibration transmissibility from the pedal to the three joints of the lower limb (ankle, knee and hip). This response is analyzed taking into account the four phases of the crank cycle: downstroke, bottom dead center, upstroke and top dead center. The downstroke begins as the foot and pedal move from 20 to $145^{\circ}$. The bottom dead center is the sector immediately following the downstroke in which the joints make a transition between extension and flexion. In the upstroke the system is pulled upward from 215 to $325^{\circ}$. The top dead center is the last transitional movement and precedes the top dead center by pressing forward over the top from about 325 to $20^{\circ}$, in a transition from flexion to extension.

The muscular activity is measured for three muscles of the lower limb.

\section{Materials and methods}

\subsection{Subject}

One healthy male cyclist $(1.75 \mathrm{~m}, 55.3 \mathrm{Kg}, 9.07 \%$ body fat, 23 years old), without apparent physical contraindications to WBV training, participated voluntarily in this study. The exclusion criteria were implants, recent fractures, musculoskeletal disorders, kidney stones, diabetes, cardio- vascular disease, gallstones or epilepsy. The subject gave a signed informed consent, and the study was approved by the local Ethics Committee.

\subsection{Material}

The test bench is composed of a vibration plate, a road bike and a power control system (Fig. 1). The vibration plate (FITVIB 600. Vreden, Germany) is equipped with two motors generating a periodic sinusoidal vibration. The vertical plate's displacement is $2 \mathrm{~mm}$ in low amplitude and $4 \mathrm{~mm}$ in high amplitude. This displacement is constant of the frequency range. The vibration plate generates a vibration in a frequency range between 20-70 Hz. The used cycle features a carbon mono-frame, Mavic Kyrium Equipe wheels, Michelin Lithion 700-23c tires (Pressure $=7$ bars). The rear wheel is placed in a Tacx Power Back home trainer. This system allows a visualization of the speed and a definition of the power that is kept constant during all tests.

Muscular activity is measured using Trigno wireless sensors (dimensions: $37 \times 26 \times 15 \mathrm{~mm}$ ) which consist of two dry bar electrodes $(10.0 \times 1.0 \mathrm{~mm})$ spaced at $10 \mathrm{~mm}$ (Delysis Inc, USA). The sensor, which allows a single differential detection of EMG signal, is characterized by a preamplifier gain of $10 \mathrm{~V} / \mathrm{V}$, noise less than $1.5 \mu \mathrm{V}$ RMS and input impedance greater than $1015 \Omega$.

Accelerometric data were obtained using an acquisition system (OROS OR35, Grenoble, France, 8 dynamic inputs, and 2 generator channels), and tri-axial accelerometers (Bruel \& Kjaer deltatron 4521B, frequency range: $0.25-3000 \mathrm{~Hz}$, sensitivity: $100 \mathrm{mV} \cdot \mathrm{g}^{-1}$ ).

Through an analog output from the OROS system, synchronization between the EMG acquisition software and the acceleration data is possible.

\subsection{Experimental protocol}

The subject wore the same shoes in both tests, to avoid external variance in the measures. Toe clips pedals were used throughout all assessments. During all the conditions tested, the subject was instructed to keep a constant position of the hands over the handlebar grip.

Muscular activity was measured for three muscles of the lower limb: Vastus Medialis: VM; Biceps Femoris: BF; Soleus: SOL (Fig. 2). Electrode skin areas were shaved, rubbed with an abrasive paste, and cleaned with alcohol solution. The EMG sensors were attached to the skin with a double-sided adhesive interface tailored to match 


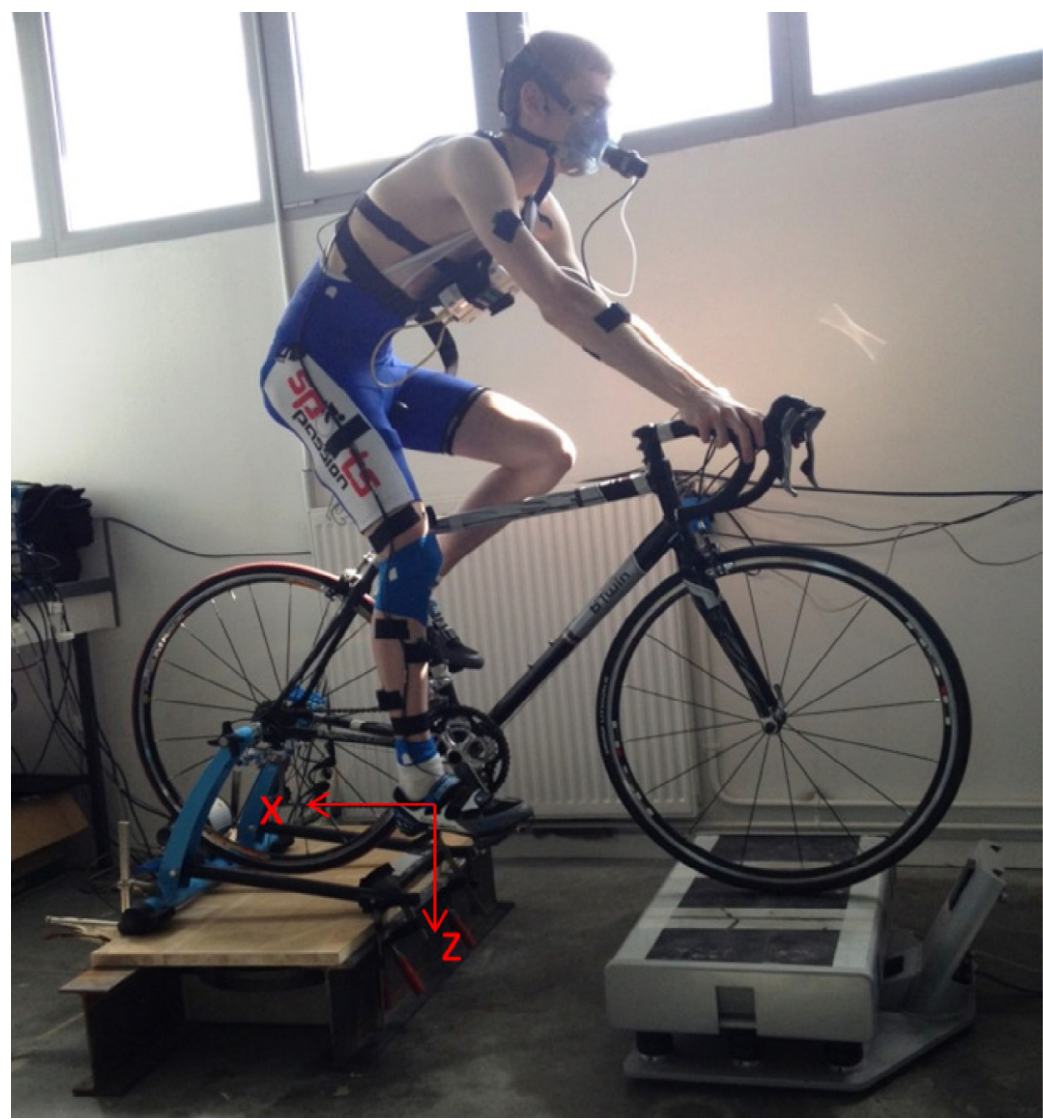

Fig. 1. Test Bench.
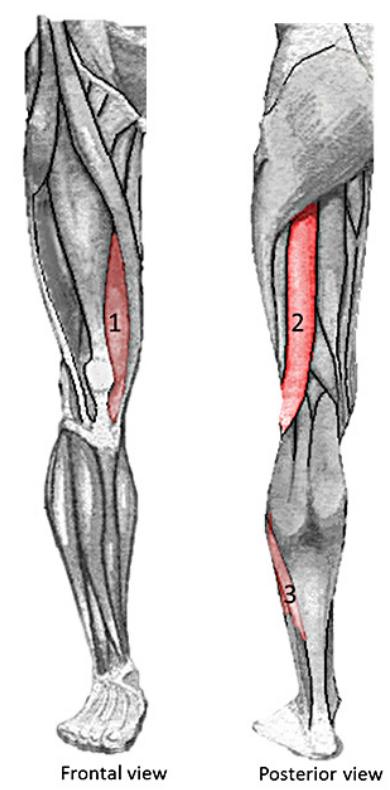

Fig. 2. Muscles Vastus Medialis (1), Biceps Femoris (2) and Soleus (3).

the contours of the sensor, and were placed on the middle of the muscle's belly and arranged in the direction of the muscle fibers, following the recommendations of De Luca et al. [14]. All the sensors were secured with specific adhesive tape to prevent their falling off due to vibrations stimulus.

The accelerometers were attached to the ankle, knee and hip regions using elastic bandages. The accelerometers were located on the skin above the lateral malleolus of the tibia (ankle), lateral tuberosity of the tibia (knee), and the anterior superior iliac spine (hip). Also, one accelerometer was attached to the pedal to provide measure of the input vibration. This value was used as a reference to compare the signals from the different body sites. All accelerometric data were sampled to $1000 \mathrm{~Hz}$ according to the Nyquist criteria.

In all the conditions, the subject was instructed to perform six minutes of a cycling at a constant speed (80 RPM) and a constant power $(150 \mathrm{~W})$, followed by four minutes of recovery time. The time of the test was chosen in order to have stabilization on the physiological parameters measured. The recovery time is used to decrease the possibility of muscle fatigue.

The exercise was repeated 16 times: for each amplitude, six conditions with vibration exposure at a frequency of 20-30-40-50-60-70 Hz and two without vibration, at the beginning of the test and in the middle of the other conditions. The data were collected over a total of 16 trials. Trials with vibration were randomized to negate any order effects. 


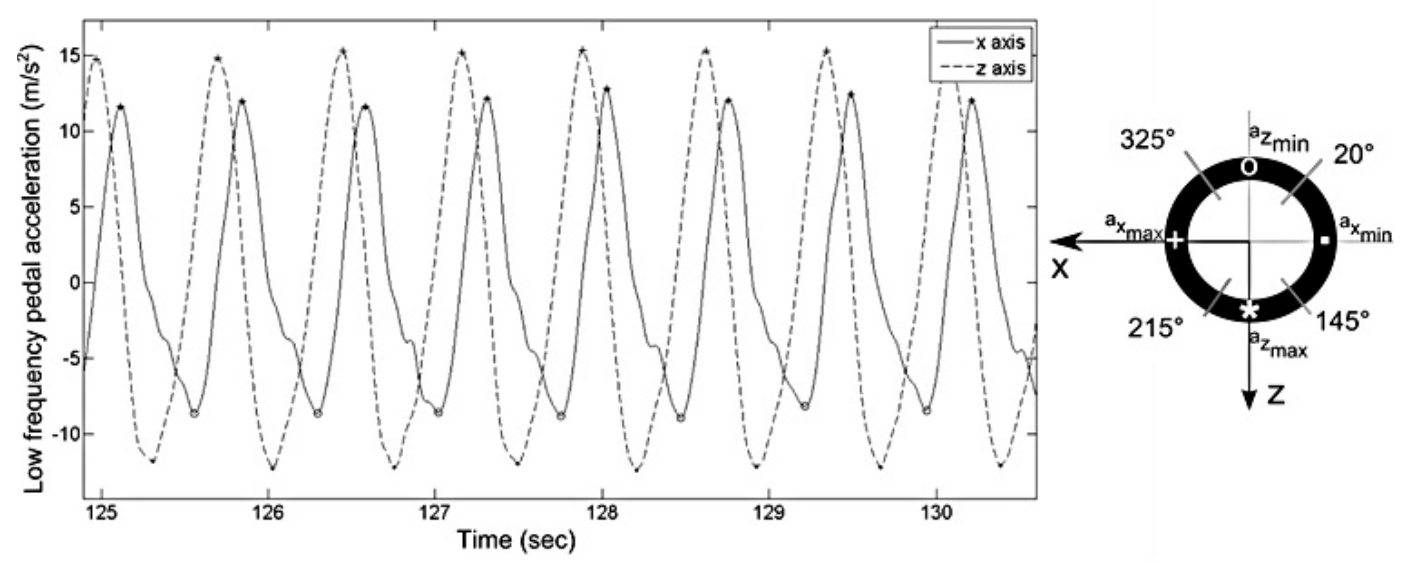

Fig. 3. Low frequency pedal acceleration in the $z$ and $x$ axis and crank cycle identification.

\subsection{Data analysis}

\subsubsection{Accelerations}

Data analysis was performed using MATLAB version 7 (The Mathworks Inc., Natick, MA). In all conditions of frequency and amplitude, the root mean square (RMS) value of the acceleration is calculated in stationary conditions. The signal acquired by the accelerometers contains a low-frequency component corresponding to the movement and a high-frequency component corresponding to the vibration. The low frequency-component is decoupled using a low pass Butterworth filter of eighth order with a cut frequency of $15 \mathrm{~Hz}$. This was carried out to remove the vibration component.

Using the raw signal, the high frequency component is filtered using a high pass Butterworth filter of eighth order with a cut frequency of $15 \mathrm{~Hz}$. This filtered signal is cut into four phases in the pedaling cycle. The cut point of those different parts is identified using the maximal acceleration in the $x$ and $z$ axes of the movement obtained with the low-frequency component in the pedal (see Fig. 3). The RMS value corresponding to each part is computed within a minute. In a minute of acquisition the measure contains approximately 80 pedaling cycles, thus the RMS value $\left(a_{\text {joint }}(f)\right)$ used to calculate the transmissibility is the mean of those RMS values corresponding to the same part of the pedaling cycle. This RMS value is calculated with the measures of the three axes using Equation (1).

For a given test frequency $f$ the accelerations $a_{\text {joint }}(f, p h)$ at each joint and each phase of the cycle $p h$, are computed using the measure in the three axes at each condition $\left(a_{\text {joint }_{x}}(f, p h) a_{\text {joint }_{y}}(f, p h), a_{\text {joint }_{z}}(f, p h)\right)$ (Eq. (1)). This acceleration is expressed in terms of transmissibility to each joint $\left(T_{\text {joint }}(f, p h)\right)$ relative to acceleration value at the input (pedal) $\left(a_{\text {input }}(f, p h)\right)$, using Equation (2). The vibration transmissibility $T_{\text {joint }}$, is studied according to the movement represented as the phase of the cycle where it is measured $(p h)$, and the excitation frequency of the vibration $(f)$.

$$
\begin{aligned}
a_{\text {joint }}(f, p h) & =\sqrt{a_{\text {joint }_{x}}^{2}+a_{\text {joint }_{y}}^{2}+a_{\text {joint }_{z}}^{2}} \\
T_{\text {joint }}(f, p h) & =\frac{a_{\text {joint }}(f, p h)}{a_{\text {input }}(f, p h)}
\end{aligned}
$$

\subsubsection{Muscular activity}

The sEMG signals were amplified using a 16-bit multi-channel, isolated biomedical signal amplifier and acquisition system (AD Instruments, Australia, PowerLab 16/35; input impedance: $1 \mathrm{M} \Omega @ 100 \mathrm{pF}$; CMRR > $120 \mathrm{~dB} @ 100 \mathrm{~Hz}$ ), sampled at $1000 \mathrm{~Hz}$. Using Matlab the signal is filtered using a band-pass filter $(10-400 \mathrm{~Hz})$ and notch filters at the frequency of the vibration exposure were used to suppress artifacts due to vibration. The RMS value of the signal at each test frequency and amplitude condition is calculated in stationary conditions. The RMS value for the sEMG signals for each condition $\left(E M G_{\mathrm{RMS}}(f)\right)$ of vibration is compared with the value assessed without vibration $\left(E M G_{-} W V_{\mathrm{RMS}}\right)$. Thus, the EMG signals are represented for each frequency as an augmentation percentage relative to the movement without vibration exposure (Eq. (3)).

$$
\begin{aligned}
& \text { Relative difference } G M(f) \\
& \qquad=\left(\frac{E M G_{\mathrm{RMS}}(f)-E M G_{-} W V_{\mathrm{RMS}}}{E M G_{-} W V_{\mathrm{RMS}}}\right) \times 100
\end{aligned}
$$

\section{Results and discussion}

\subsection{Transmissibility}

The mean and standard deviation over a minute of the transmissibility from the pedal to the ankle (Fig. 4), knee (Fig. 5) and hip (Fig. 6) are calculated. This transmissibility varies depending on the frequency and the amplitude of the vibration exposure, as well as the analyzed pedaling phase and the joint where it is measured. 
a)

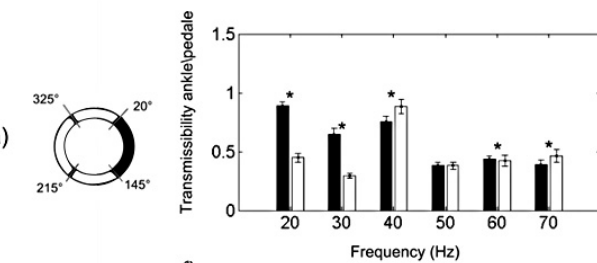

c)

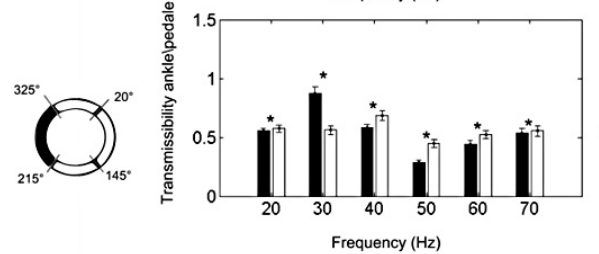

b)

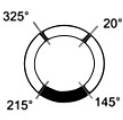

d)

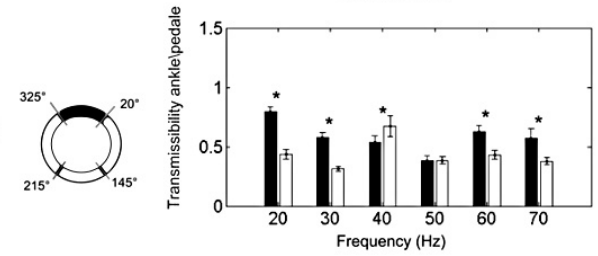

Fig. 4. Ankle/Pedal transmissibility for low at high amplitude at the four phases of the pedaling cycle. (a) Phase 1: downstroke $\left(20^{\circ}-145^{\circ}\right)$, (b) Phase 2: bottom dead center $\left(145^{\circ}-215^{\circ}\right)$, (c) Phase 3: upstroke $\left(215^{\circ}-325^{\circ}\right)$, (d) Phase 4: top dead center $\left(325^{\circ}-20^{\circ}\right)$. $*$ Significant difference between vibration transmissibility at low and high amplitude vibration $(p<0.05)$.

a)

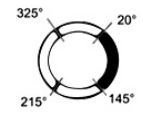

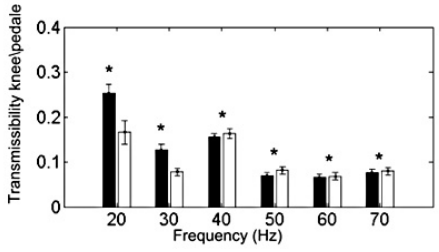

c)

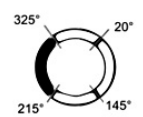
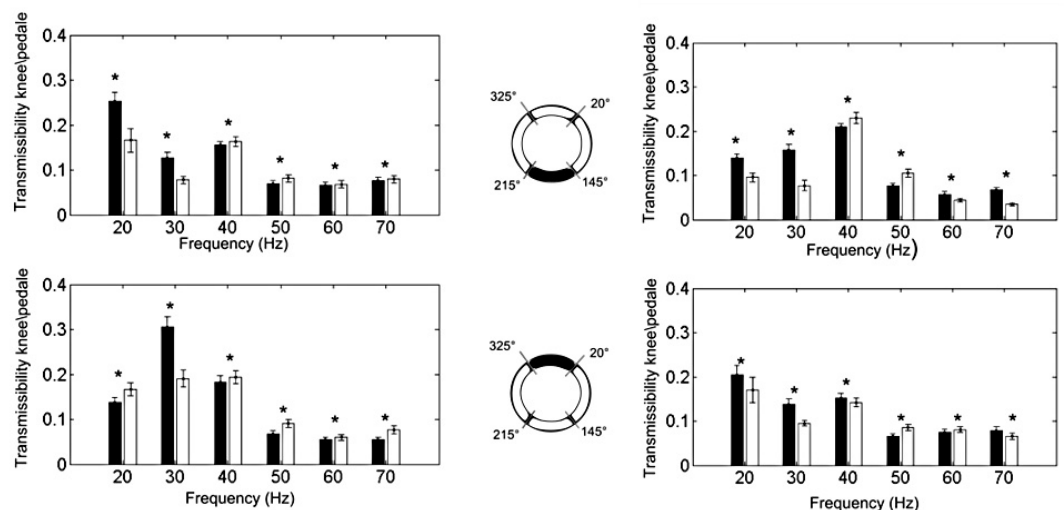

Low amplitude High amplitude
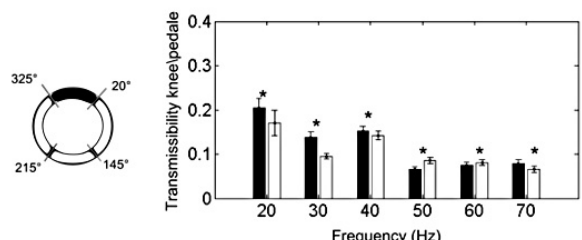

Fig. 5. Knee/Pedal transmissibility for low at high amplitude at the four phases of the pedaling cycle. (a) Phase 1: downstroke $\left(20^{\circ}-145^{\circ}\right)$, (b) Phase 2: bottom dead center $\left(145^{\circ}-215^{\circ}\right)$, (c) Phase 3: upstroke $\left(215^{\circ}-325^{\circ}\right)$, (d) Phase 4: top dead center $\left(325^{\circ}-20^{\circ}\right)$. Significant difference between vibration transmissibility at low and high amplitude vibration $(p<0.05)$.

a)<smiles>Brc1ccccc1</smiles>

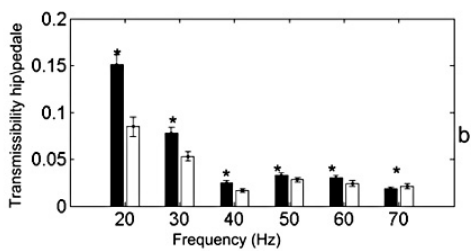

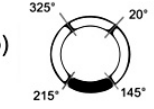

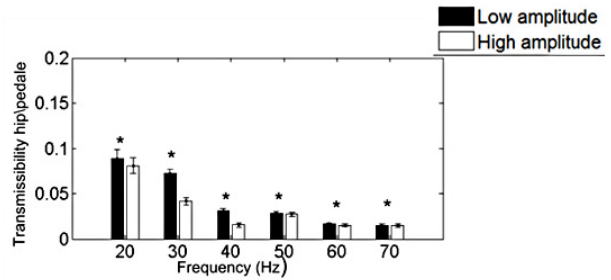

c)<smiles>Brc1ccccc1</smiles>

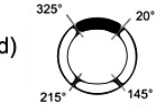

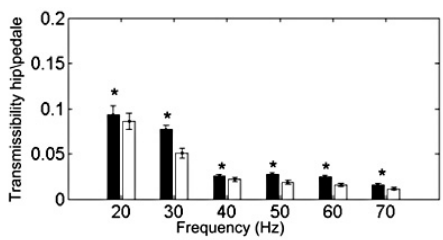

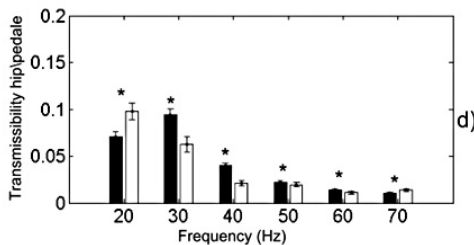

Fig. 6. Hip/Pedal transmissibility for low at high amplitude at the four phases of the pedaling cycle. (a) Phase 1: downstroke $\left(20^{\circ}-145^{\circ}\right)$, (b) Phase 2: bottom dead center $\left(145^{\circ}-215^{\circ}\right)$, (c) Phase 3: upstroke $\left(215^{\circ}-325^{\circ}\right)$, (d) Phase 4: top dead center $\left(325^{\circ}-20^{\circ}\right)$. ${ }^{*}$ Significant difference between vibration transmissibility at low and high amplitude vibration $(p<0.05)$. 


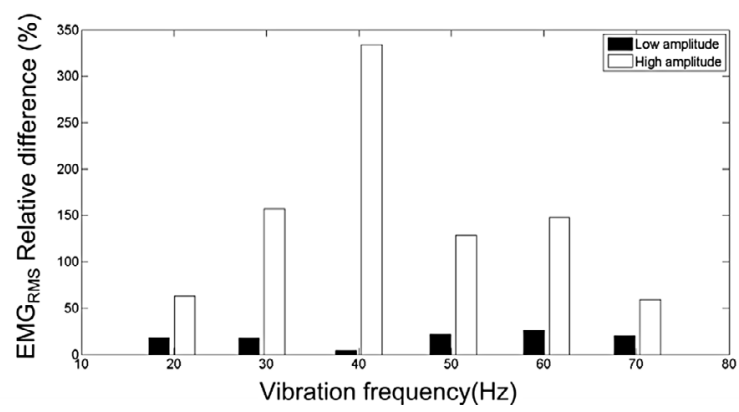

Fig. 7. Relative difference in the $\mathrm{EMG}_{\mathrm{RMS}}$ value for the muscle Soleus between cycling with and without vibration.

The vibration transmissibility for the ankle joint ranges between 0.28 and 1.10 . The ankle joint presents an amplification of the input acceleration (1.1 times) only at $40 \mathrm{~Hz}$ in the phase 2 , which is the phase of transition between flexion and extension. This joint shows attenuation in the other frequency conditions; however, at low frequencies between 20 and $40 \mathrm{~Hz}$ the transmissibility is close to 1 . That means that the vibration measured in the joint is almost the same that the vibration in the pedal.

Compared to the ankle, the knee and hip joints never present an amplification of the vibration. For the knee the vibration transmissibility ranged between 0.03 and 0.35 . The resonance peak in the knee and the hip occurs at low frequencies $(20-30 \mathrm{~Hz})$. In the knee the maximal vibration transmissibility is 0.35 and for the hip is 0.15 . In the hip joint, the vibration is largely attenuated in all the studied conditions (0.01-0.15)

The significant difference between the vibration transmissibility at low and high amplitude for each frequency was evaluated using a Mann Whitney statistical test. This difference $(p<0.05)$ is shown in the figures. At the knee and hip joint a significant difference between low and high amplitude is found for all the studied conditions in frequency and pedaling phase. At the ankle joint this difference is found for all the conditions, except for $50 \mathrm{~Hz}$ at the phases 1 and 4, when the ankle is in extension. At the ankle joint, lower amplitude presents a higher transmissibility at low frequencies $(20-30 \mathrm{~Hz})$ while it presents a higher transmissibility with higher amplitude at higher frequencies (40-50-60-70 Hz).

The transmissibility at the joints decreased with distance from the pedal. This fact shows a damping behavior of the tissues in the lower limb, explained by a larger quantity of body's natural shock absorbers (muscles, bone and ligaments) present at more proximal locations of the lower limb.

\subsection{EMG}

The RMS value of the sEMG was calculated over a minute for each condition, and represented as relative difference (\%) between the cycling exercise with and without vibration for the muscles Soleus (SOL) (Fig. 7), Vastus Medialis (VM) (Fig. 8) and Biceps Femoris (BF) (Fig. 9).

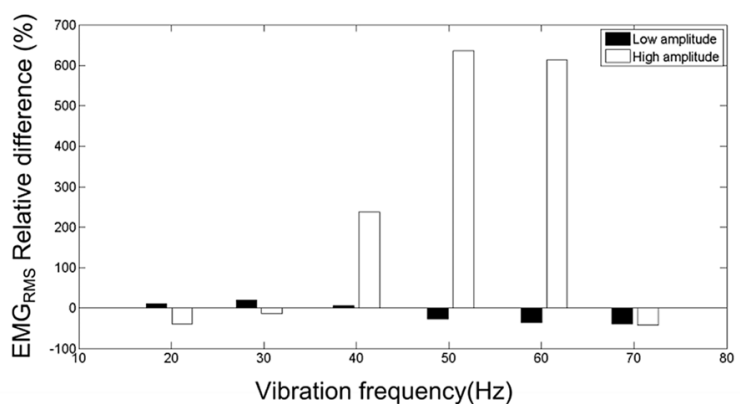

Fig. 8. Relative difference in the $\mathrm{EMG}_{\mathrm{RMS}}$ value for the muscle Vastus Medialis between cycling with and without vibration.

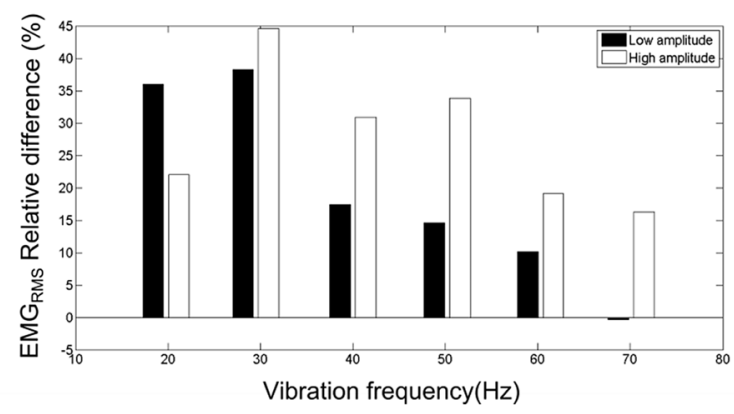

Fig. 9. Relative difference in the $\mathrm{EMG}_{\mathrm{RMS}}$ value for the muscle Biceps Femoris between cycling with and without vibration.

For the muscle SOL the increase due to vibration is always much higher at high amplitude than at low amplitude vibration. This increase has a peak at $40 \mathrm{~Hz}$ for high amplitude vibration, where the increase of the muscle activity is $330 \%$. This muscle is located in the superficial posterior region of the lower leg, and its action is plantarflexion of the foot (ankle extension). The peak found for the muscular activation in high amplitude vibration seems to be related to the transmissibility in the ankle, because they are both found at the same frequency (40 Hz).

For the muscle VM, the increase in the activity for high amplitude vibration at 40,50 and $60 \mathrm{~Hz}$ is much higher than the increase for the other studied muscles. This increase has a peak at $50 \mathrm{~Hz}$ where the muscular activity is increased in a $636 \%$ compared to the exercise without vibration.

The muscle BF presents an increase of the muscular activity for almost all the conditions, except for low amplitude vibration with frequency of $70 \mathrm{~Hz}$ where the activity suffers a very small decrease (less than $5 \%$ ). This increase is maximal for high amplitude vibration at $30 \mathrm{~Hz}$ with a value of $45 \%$. At $20 \mathrm{~Hz}$ low amplitude vibration presents a higher increase in the activity compared to high amplitude vibration. However, for the frequencies greater than $30 \mathrm{~Hz}$ high amplitude vibration presents a higher increase which declines when the frequency increases.

The BF is a muscle of the posterior thigh, its action is knee flexion and it is also involved in hip extension. 
The behavior of this muscle's muscular activation increase seems to be related to the transmissibility at the hip, showing a peak in low frequencies $(20-30 \mathrm{~Hz})$ and a decrease with the increase of the frequency above $40 \mathrm{~Hz}$.

The increase in the $E M G_{\mathrm{RMS}}$ of this muscle is lower than for the other two studied muscles, this could be explained by the greater distance from this muscle to the source of vibration (pedal). As the distance from the pedal increases, the transmissibility decreases; so, this could cause also a smaller change in the muscular activation relative to the exercise without vibration.

\section{Conclusions}

The protocol developed in this article, shows the possibility of analyzing simultaneously the dynamic and physiological response of the human body under vibration in cycling.

The preliminary results of the transmissibility allow one to identify the resonance frequency at each joint for every tested condition. As shown by Chiementin et al. [6] the frequency of vibration exposure in cycling is related to the displacement speed. Thus, the identified resonance frequencies represent different displacement speeds where the acceleration received by the joint is higher than the vibration entering the lower limb system. It is important to highlight that the increase in this acceleration can lead to joint damage [15]. Using the low frequency component of the pedal acceleration, it is possible to identify the different parts of the crank cycle and to calculate the transmissibility value in those parts, revealing the influence of the movement on this value.

The EMG signals are modified by the vibration exposure in almost all the tested conditions. The changes in EMG signals seem to be related to the transmissibility at the joint with which the muscle is associated. However, the EMG signals in this preliminary study generally present a much higher difference between low and high amplitude vibration than the vibration transmissibility.

Due to the high variability in measures relative to the human body, and particularly the measures of muscular activation by EMG a number of subjects is necessary to test the hypothesis here exposed in order to use statistical test.

Acknowledgements. These investigations are financially supported by the Champagne-Ardenne region and by The European Found of Regional Development (FEDER). The authors wish to thank neXXtep Technologies for their contribution.

\section{References}

[1] A. Peretti, L. Pignalosa, F. Bonomini, A. Paoli, G. Bartolucci, Vibrazioni su biciclette da corsa e da città, G. degli Ignienisti Ind. 34 (2009) 283-293

[2] J. Parkin, E. Sainte Cluque, The impact of vibration on comfort and bodily stress while cycling, in UTSG 46th Annual Conference, Newcastle University, 2014, pp. 6-8

[3] F. Giubilato, N. Petrone, A method for evaluating the vibrational response of racing bicycles wheels under road roughness excitation, Procedia Eng. 34 (2012) 409-414

[4] M. Olieman, R. Marin-Perianu, M. Marin-Perianu, Measurement of dynamic comfort in cycling using wireless acceleration sensors, Procedia Eng. 34 (2012) 568573

[5] C. Hölzel, F. Höchtl, V. Senner, Cycling comfort on different road surfaces, Procedia Eng. 34 (2012) 479-484

[6] X. Chiementin, M. Rigaut, S. Crequy, W. Bertucci, Hand-arm vibration in cycling, J. Vib. Control (2011)

[7] N. Petrone, F. Giubilatoa, Development of a Test Method for the Comparative Analysis of Bicycle Saddle Vibration Transmissibility, in 6th Asia-Pacific Congress on Sports Technology (APCST) (2013) 288-293

[8] M. Munera, X. Chiementin, S. Crequy, W. Bertucci, Physical risk associated with vibration at cycling, Mech. Ind. 15 (2014) 535-540

[9] M.J. Griffin, Handbook of Human Vibration, 1996 , p. 1008

[10] B. Sperlich, H. Kleinoeder, Physiological and perceptual responses of adding vibration to cycling, J. Exerc. Physiol. 12 (2009) 40-46

[11] B. Samuelson, L. Jorfeldt, B. Ahlborg, Influence of vibration on work performance during ergometer cycling, Ups. J. Med. Sci. 94 (1989) 73-9

[12] P. Arpinar-avsar, G. Birlik, Ö.C. Sezgin, A.R. Soylu, The Effects of Surface-Induced Loads on Forearm Muscle Activity during Steering a Bicycle, 2013, pp. 512-520

[13] J. Srinivasan, V. Balasubramanian, Low back pain and muscle fatigue due to road cycling-An sEMG study, J. Bodyw. Mov. Ther. 11 (2007) 260-266

[14] C.J. de Luca, P.J. Foley, Z. Erim, Motor unit control properties in constant-force isometric contractions, J. Neurophysiol. 76 (1996) 1503-16

[15] S. Carlso, The effect of vibration on the skeleton, joints and muscles.pdf, Appl. Ergon. 13 (1982) 251-258 